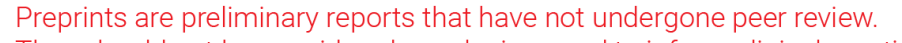 They should not be considered conclusive, used to inform clinical practice, or referenced by the media as validated information. \\ Exploration of Key Genes Combining with Immune Infiltration Level and Tumor Mutational Burden in Hepatocellular Carcinoma
}

Jing Chen ( $\sim$ jingguogu0960926@163.com )

Affiliated Hospital of Nantong University

shuzhen wu

Affiliated Hospital of Nantong University

sijia ge

Affiliated Hospital of Nantong University

ran Ji

Affiliated Hospital of Nantong University

\section{Yuyan Chen}

Affiliated Hospital of Nantong University

Wei Huang

Affiliated Hospital of Nantong University

Cui-hua Lu

Affiliated Hospital of Nantong University

\section{Research}

Keywords: Hepatocellular carcinoma, Tumor mutational burden, Tumor immune infiltration, prognosis.

Posted Date: August 17th, 2021

DOl: https://doi.org/10.21203/rs.3.rs-798919/v1

License: (9) This work is licensed under a Creative Commons Attribution 4.0 International License.

Read Full License 


\section{Abstract}

Background: Hepatocellular carcinoma (HCC) is still a lethal malignancy because of its heterogenicity and aggressive behavior, with unsatisfactory early diagnosis and poor prognosis. Recently, few somatic mutations have been reported to associated with HCC carcinogenesis and function in predicting HCC progression. Interactions of tumor cells with surrounding immune microenvironment in HCC participate in orchestrating the onset and development. Herein, our aim is to investigate the associations of tumor mutational burden (TMB) with immune microenvironment in HCC. Then we will seek for differential expression genes (DEGs) in terms of immune and TMB scores and discuss whether their latent functions affect HCC prognosis and progression.

Methods: The expression, clinical and mutational data were downloaded from TCGA database, then calculated the immune infiltration of these HCC samples by R package "SSGSEA", "CIBERSORT" and "ESTIMATE". Then the samples were clarified to 2 groups according to the immune levels. TMB was also calculated and differential expressional genes (DEGs) in the low and high TMB group were intersected and the different immune level groups. Then the cox analyses and prognostic model were performed and tested by R package "glmnet". Then the selected genes BCL10 and TRAF3 were tested their expression by qrt-PCR and IHC and tested their clinical correlation by chi-square analyses and their biological processes enriched by GSEA, and their immune infiltration by "SSGSEA" individualy. Last, pearson algorithm was employed to judge the relevance of BCL10 and TRAF3.

Results: Upregulated degrees of immune infiltration correlated with TMB, they synergistically predicted poor prognosis in HCC. DEGs enriched in immune-related pathways could serve as an indicator of therapeutic effect of HCC immunotherapy. Among these DEGs, BCL10 and TRAF3 were highly expressed in HCC tissues, especially in TP53 mutation group. BCL10 and TRAF3 corporately exhibited immunological function, thereby affecting HCC progression and prognosis.

Conclusions: We identified that BCL10 and TRAF3 exhibited good prognostic value in predicting the clinical outcome of HCC patients, which may influence TMB and tumor microenvironment (TME) and help us moving towards immune-based therapies with HCC patients, ultimately improving their long-term survival.

\section{Introduction}

Liver hepatocellular carcinoma (LIHC) occupies nearly 90 percent of primary hepatic carcinoma possesses high morbidity and mortality, which is the fifth most prevalent malignancies and the second primary cause of cancer -related death globally[1]. World Health Organization speculated that in 2030, about one million patients with hepatopathy will die from hepatocellular carcinoma[2]. LIHC is attributable to chronic hepatitis or cirrhosis arise from various etiologies, including infection with hepatitis B and C virus, as well as alcoholic and nonalcoholic fatty hepatic disease[3]. Satisfactory sanative therapies for hepatocellular carcinoma (HCC) comprised of surgical resection, 
chemoradiotherapy, ablation, immunotherapy and liver transplantation[4]. However, the treatment of HCC is still challenging owing to the lack of early diagnosis, potent prognosis markers and its high recurrence rate and metastasis[5]. Thus, discovering reliable molecular mechanisms for HCC is imminently needed to improve long-term survival of patients.

Gene mutations are abrupt, heritable variations in genomic DNA molecules. Mutants and viruses in the environment stimulate the activation of hepatocyte division response pathway, causing cell point mutation and gene translocation, which accelerate the malignant transformation of cancer cells[6]. Tumor mutational burden (TMB) is defined as the total amount of somatic mutations per megabase (Mb) within the tumor genomic sequence [7]. These genetic mutations include nonsynonymous mutations mostly comprised of missense mutations. In addition, synonymous mutations, insertions or deletions and copy number gains and losses are also included in some cases[8]. Recently, TMB is considered to serve as a biomarker across diverse solid cancers for prediction of the neoantigen load, tumor immunogenicity and prognosis after clinical treatment[9]. Interestingly, TMB levels in patients varies in a tumor type dependent manner, thus contribute to opposing prognosis and survival influences across tumor types[10, 11]. Considering that the heterogeneity between individual tumors, the frequency of TMB can vary across tumor types[12]. Therefore, it is high time to take tumor/microenvironment interaction into account when analyzing the correlation of TMB with prognosis. Nonetheless, the value of TMB is still unclear and no specific relationship has been uncovered between TMB and prognosis in patients with HCC, which need further elucidation.

Currently, immune-related TME has been recognized to exert a pivotal influence during tumor progression[13]. Immune infiltration in TME has been emphasized by previous researches to be responsible for the early recurrence and metastasis in HCC[14]. Tumor-infiltrating immune cells are reported to contribute to impaired anti-tumor immunoreaction in response to disordered metabolic activity of tumor cells, which is speculated to participate in immune suppression and malignant progression of HCC[15]. Notably, immunotherapy such as immune checkpoint inhibitor (ICI) is recognized as a therapy for various malignancies[16]. Additionally, TMB has been discovered to closely correlated to immunotherapy in several cancer types. The impact of TMB on prognosis and its connection with immune infiltration varied across different cancer types[17]. Because few studies had concentrated on TMB and immune infiltration with respect to $\mathrm{LIHC}$, so we conducted this research to inquire into the prognostic value of TMB and its latent correlation with immune infiltration in LIHC.

In our study, we use databases to evaluate the different degrees of immune infiltration on prognosis and mutation in LIHC. In addition, the correlation between TMB and immune infiltration and their jointly predictive value were also studied by using multiple bioinformation analysis methods. Next, we selected different expression genes based on immune and TMB score in $\mathrm{HCC}$ and performed functional enrichment and univariate Cox regression analysis. A prognostic signature was also constructed and the superiority of the model was assessed. Then, we found that BCL10 and TRAF3 expression were evidently elevated in TP53 mutation group in HCC tissues and were associated with several clinical prognostic factors. Finally, functional enrichment, immune infiltration and correlation analysis of BCL10 and TRAF3 
were performed. Therefore, BCL10 and TRAF3 are involved in TMB and tumor immune microenvironment, thus influencing the progression and prognosis of HCC.

\section{Methods}

\subsection{Patients and sample collection}

This study was approved by the ethics committee of the affiliated hospital of Nantong University (AHNTU). Total HCC tissues $(n=20)$ and surrounding normal tissues $(n=20)$ were obtained from the Affiliated Hospital of Nantong University for IHC and qRT-PCR. Permission was approved by the Human Research Ethics Committee of AHNTU, and written informed consent was obtained from each patient.

\subsection{Data downloading and processing from TCGA LIHC cohort}

Transcriptional data, including the counts data and FPKM data, and correspondingly clinical data, including overall survival status, overall survival time, $T$ stage, $\mathrm{N}$ stage, $\mathrm{M}$ stage and Ajcc stage from TCGA LIHC cohort were obtained by the R package "TCGAbiolinks"[18]. Somatic mutation data from TCGA LIHC cohort were obtained with the selected "mutect2" dataset and visualized by the R package "maftools"[19].

\subsection{Tumor Immune microenvironment purity assessment}

Implementation of Single-Sample Gene Set Enrichment Analysis (ssGSEA)[20], CIBERSORT algorithm[21], ESTIMATE algorithm were facilitated to evaluate the immune infiltration subsets of each sample individually. A reference document containing the key gene expression of 22 leukocyte subtypes were supplemented in the CIBERSORT algorithm.

\subsection{Consensus clustering Analyzing}

The 374 HCC specimens from TCGA LIHC cohort were classified into 2 clusters by the R package "ConsensusClusterPlus" [22] according to the immune infiltration level from CIBERSORT.

\subsection{Tumor mutational burden (TMB) calculating}

TMB function as the mature biomarkers in immune checkpoint inhibitors, which is considered to directly measure the number of mutations in tumor tissues. Here, we selected the calculating methods recently reported by Zachary[23]. After assessing the TMB score for each sample in TCGA LIHC dataset, the samples were divided to the high TMB score and the low TMB score group, for subsequent detecting of the differential expressed genes (DEGs).

\subsection{DEGs and enrichment measurements}

Under the screening criteria of DEGs: |log2Foldchange| $>1$, adjust $P$ value $<0.05$, R package "Deseq2" was utilized to get the DEGs between the ICR1 and the ICR2 cluster, and between the different levels of TMB 
group[24]. 369 genes in total were obtained from these two differential analyses. Then gene ontology (GO), including the MF, BP, CC pathways and Kyoto Encyclopedia of Genes and Genomes (KEGG) pathway enrichment were employed to analyze underlying biological characteristics and significance of theses DEGs[25].

\subsection{Single-gene for Gene Set Enrichment Analysis (GSEA)}

With the purpose of studying possible pathways associated with BCL10 and TRAF3 from the TCGA_LIHC dataset, respectively, GSEA generated an ordered list of all genes from TCGA LIHC FPKM transcriptional data based on their correlation with BCL10 and TRAF3 expression, respectively. The "h.all.v6.2.entrez.gmt" were chosen as reference gene sets. Then, the distinction in gene enrichment between the high-level BCL10 and TRAF3 group and the low-level BCL10 and TRAF3 group were analyzed using GSEA Version 2.10.1 software[26].

\subsection{Prognostic model construction and inspection}

First, univariate Cox regression model was constructed to analyze the 369 DEGs which can predict the prognosis of HCC patients and the visualized forest plot manifested the results. Then the LASSO Cox regression model was constructed and fitted with by R package "glmnet"[27]. Under this progression, 19 key genes were concluded in the formula to calculate the risk scores (RS). Then we generated ROC curve by "ROCR" to assess the efficiency of RS to predict prognosis[28]. In order to better predict the prognosis of HCC patients by combining our predicted RS with clinical indexes, Nomogram was integrated with RS, T stage, $\mathrm{N}$ stage, $\mathrm{M}$ stage and age, gender and generated[29]. Lastly, C-Index, Net reclassification index (NRI) and Decision Curve Analysis (DCA) were calculated to evaluate the nomogram.

\subsection{RNA extraction and quantitative real time-polymerase chain reaction (qRT-PCR)}

We carried out RNA extraction and qRT-PCR procedure as previously depicted [30]. The BCL10 and TRAF3 primer sequences are listed below.

BCL10 Forward: 5'-ACCTCACTGAAGTGAAGAAG-3'; BCL10 Reverse: 5'-ATCTGGAGAGGTTGTTCGTG-3'; TRAF3 Forward: 5'-TGAGCTGGAGAGCGTAGACA-3'; TRAF3 Reverse: 5'-AGATCAGCACCCCGTTGTAG-3'; GAPDH Forward: 5'-AGAAGGCTGGGGCTCATTTG-3'; GAPDH Reverse: 5'-AGGGGCCATCCACAGTCTTC-3'.

\subsection{Immunohistochemistry (IHC)}

Briefly. Paraffin-embedded slides were incubated with BCL10 (Abcam, Burlingame, CA, USA, 1:100) and TRAF3 (Abcam, 1:100). Avidin-biotin-peroxidase complex and DAB substrate were used for the detection of primary antibody. Anti-digoxigenin-HRP (anti-DIG HRP) antibody and DAB were used for detection. Then the slices were dehydrated, cleared and photographed by Olymbus light microscope.

\subsection{Statistical analysis}


Statistical data analyses were all implemented and generated through $\mathrm{R}$ software 4.0.3, all the plots were generated by R package "ggplot2"[31]. The value of all experiments were quantified by mean \pm SD. The correlation coefficient between two groups (BCL10/TRAF3) of HCC and pan-cancer samples in TCGA was generated by Pearson's correlation analysis. $P$ values less than 0.05 was considered statistically significant.

\section{Results}

\subsection{The landscape of immune infiltration in HCC patients from TCGA}

In order to study the immune infiltration of liver cancer patients, we facilitated the data from TCGA LIHC dataset and performed CIBERSORT to quantify the immune cells levels of HCC. A landscape of immune cell interaction in HCC was illustrated by the correlation coefficient heatmap (Fig. 1A). In order to understand whether the samples could contribute to the degree of immune infiltration, we sought the consensus clustering analysis to stratify 374 samples, which were divided into 2 groups according to optimal cluster number $(k=2)$ (Fig. 1B). Then ssGSEA was used and we found that the immune cluster 1(ICR1), which was defined as the high immune infiltration, was linked to activated B cell, activated CD4 T cell, activated CD8 T cell, activated dendritic T cell, century memory CD8 T cell, effector memory CD4 T cell, effector memory CD8 T cell, Gamma delta T cell, Immature B cell, Immature dendritic cell and macrophage compared with the ICR2 group (Fig. 1C, P<0.001). Estimate scores, immune scores and stromal scores were procured via applying ESTIMATE algorithm, and they were higher in ICR1 compared with ICR2 (Fig. 1D, $P<0.001$ ). Also, patients' overall survival $(O S)$ in ICR1 group was significantly reduced compared to that of patients in ICR2 group, as visualized by Kaplan-Meier survival curves (Fig. 1E, P = 0.0079). Together, these data revealed that higher immune infiltration level was positively correlated with poor prognosis.

\subsection{Comparisons of mutation profiles in HCC}

The somatic mutation profiles of 74 HCC samples from ICR1 and 285 HCC samples from ICR2 were downloaded from TCGA. Then the results based on the mutation data were visualized in VCF format utilizing the "maftools" package. Mutation information for each gene in each sample was shown within waterfall plot, where colored annotations at the bottom signified different types of mutation (Fig. 2A, B). Interestingly, $94.59 \%$ (70 of 74 ) of patients in the ICR1 group had mutations, while $82.81 \%$ (236 of 285 ) of patients in the ICR2 group had mutations. And the mutation rate of TP53 in the ICR 1 group was 50\%, while in the ICR2 group it was $25 \%$. Thus these findings indicate that different immune infiltration levels are associated with mutations in HCC.

\subsection{The Correlation between the immune infiltration and Somatic Variants in HCC}


Tumor mutation burden (TMB) is defined as the amount of somatic gene coding errors, base substitutions, gene insertion or deletion errors detected per megabase in total. To further understand the relevance of immune infiltration degrees and mutation, we then assessed the TMB of the ICR1 and ICR2 group and we found The TMB is higher in ICR1 group than ICR2 (Fig. 3A, P<0.001). The Estimate score was subsequently verified to be positively related to the TMB in ICR1 group by using correlation analyses (Spearman coefficient: $R^{2}=0.06, p=0.0037$ ), while have no obvious correlation in ICR2 group (Spearman coefficient: $R^{2}=0.02, p=0.262$, Fig. $3 B$ ). Next, patients' overall survival (OS) in high TMB group was significantly lower than that of patients in low TMB group revealed by Kaplan-Meier survival curves (Fig. 3C, $\mathrm{P}<0.001$ ). At the same time, considering that the survival probability was lower in the ICR1 group compared to that of the ICR2 group in result 1, so we used the Kaplan-Meier survival curves and displayed great differences of survival in subgroups( High TMB \& ICR1 group versus High TMB \& ICR2 group, $p<0.001$; Low TMB \& ICR1 score group versus Low TMB \& ICR2 group, $p<0.001$; Fig. 3D). Thus these findings indicate that the high immune infiltration level might correlated with TMB and could synergize with high TMB level to predict poor prognosis.

\subsection{Differential expressed genes with immune and TMB score in HCC}

In search of the differential expressed genes (DEGs) contribute to different immune infiltration level and TMB level. The "Limma" package was performed, and 1424 DEGs were selected in the high or low TMB score group, while 2181 DEGs were selected in the ICR1 or ICR2 group. There are also 369 DEGs in these two groups at the same time (Fig. 4A). In addition, GO functional enrichment (BP, MF and CC) (Fig. 4B-D) and KEGG pathway analyses (Fig. 4E) was conducted in 369 co-expressed DEGs. Interestingly, these genes were enriched in Positive regulation of macrophage colony - stimulating factor stimulus pathway, B cell apoptotic process pathway, T cell receptor complex pathway, B cell receptor signaling pathway, $T$ cell receptor signaling pathway. These findings suggested that these genes may function as underlying predictive indicators which can response to immunotherapy in HCC. Meanwhile, univariate Cox regression analysis of 89 significant DEGs $(P<0.05)$ from the 369 DEGs was performed in a forest plot $(F i g .4 F-H)$.

\subsection{Construction of the combined prognostic risk signature in HCC.}

Then, a generalized linear model named LASSO algorithm was carried out to build the prognostic signature. The $\log _{2}$ transformation of the lambda $(\lambda)$ value generated a coefficient profile plot, which was determined when the likelihood deviance was minimum (Fig. 5A). Nineteen DEGs were included and the formula was generated: $=-0.043$ GHR expression -0.005 RORC expression - 0.184 CHP1 expression + 0.066 HBEGF expression + 0.004 CXCL8 expression + 0.081 PGF expression + 0.027 SPP1 expression + 0.083 TXLNA expression + 0.010 CSF3R expression - 0.001 ACKR1 expression - 0.176 ENG expression + 0.138 IL 15RA expression - $0.088 C D 8 A$ expression - 0.143 ZAP70 expression + 0.056 BCL 10 expression - $0.043 C D 79 A$ expression $+0.006 C D 209$ expression +0.199 TRAF3 expression +0.317 SLC29A3 expression. Also, we calculating the corresponding coefficients using the minimum 10 -fold cross- 
validation mean square error in HCC (Fig. 5B). Each patient's risk score $=\sum$ gene expression*coefficient (glmnet R package). The results showed that compared with the surviving patients, dead patients' risk score was markedly higher (Fig. $5 \mathrm{C}, \mathrm{P}<0.001$ ). In order to judge the accuracy of the prediction results, we then generated the ROC, which was 0.722 (Fig. 5D, $p<0.001$ ). Then age, gender, $T$ stage, $N$ stage, $M$ stage and risk score were all included to establish a nomogram for predicting 1-, 2-, 3-year OS. Experimental results demonstrated that $\mathrm{T}$ stage contributed the most to prognosis, secondly was the risk score (Fig. 5E). To evaluate the constructed prognostic model, we worked out that the C-index of our nomogram was 0.736 , and Fig. $5 \mathrm{~F}$ presented the DCA plots of our nomogram. Besides, to evaluate the calibration of the nomogram, we utilized 1000 resamples boot-strapping procedure and obtained revised estimates of predicted and actual values. The calibration graphs showed remarkable accordance between predicted and actual survival rates (Fig. 5G). All these results substantiated the excellent clinical value of the nomogram compared to the risk score in predicting OS.

\subsection{Expression and clinical correlation of BCL10 and TRAF3 in HCC}

To find whether the 19 DEGs were correlated with TP53 mutation, we analyzed the data from TCGA LIHC dataset and discovered the higher BCL10 and TRAF3 expression levels in TP53 mutation group than that in the TP53 wild group (Fig. 6A-B, $p<0.001$ ). Then the scatter and box plot revealed that BCL10 and TRAF3 expression were significantly elevated in HCC tissues in the TCGA LIHC dataset (Fig. 6C-D, $p=$ $0.011 ; p<0.001$ ) and AHNTU cohorts (Fig. 6E-F, p<0.001; $<<0.01$ ). To further investigate the BCL10 and TRAF3 protein expression, IHC was performed to reveal the staining distribution in $\mathrm{HCC}$ and adjacent tissues (Fig. 6G-H). Mining the clinical data of 234 HCC patients from TCGA, the high BCL10 level was corelated with the status $(p=0.008)$, Ajcc stage $(p=0.0075)$ and T stage $(p=0.0076$, Fig. 61$)$, while the TRAF3 expression was associated with stage $(p=0.044$, Fig. $6 \mathrm{~J})$.

\subsection{Functional enrichment analysis and correlation of BCL10 and TRAF3}

For the sake of understanding the potential characteristics of BCL10 and TRAF3. GSEA analysis of each hub gene with the TCGA LIHC dataset was performed. The samples $(n=374)$ were partitioned into 2 groups on the basis of the median expression values of BCL10 and TRAF3. BCL10 and TRAF3 were all enriched in functions: apoptosis, IL-2 STAT5 signaling, P53 signaling, PI3K-AKT-MTOR signaling, TGF- $\beta$ signaling, TNFa signaling via NF-KB and inflammatory response, while negatively enriched in Bile Acid and Fatty Acid metabolism (Fig. 7A-B). Then, to validate the relationship of BCL10 and TRAF3 levels with the immune microenvironment in-depth, the ratio of immune infiltration subsets was analyzed using ssgsea and we found BCL10 and TRAF3 were all correlated with activated CD4 T cell, central memory CD4 T cell, type $2 \mathrm{~T}$ helper cell, natural killer T cell, effector memory CD4 T cell, natural killer cell, central memory CD8 T cell, plasmacytoid dendritic cell, immature B cell, T follicular helper cell, MDSC, neutrophil, activated CD8 T cell, and eosinophil cells ( $p<0.05$; Fig. 7C-D). BCL10 and TRAF3 enrich in various 
pathways in GSEA analysis hinted that there is a potential connection and correlation between BCL10 and TRAF3. Then the pearson test was used and we found BCL10 is significantly correlated with TRAF3 in $\mathrm{HCC}(r=0.65, p<0.0001$, Fig. 7E). Further, BCL10 was significantly correlated with TRAF3 in pan cancers ( $r=0.53, p<0.0001$, Fig. 7F), while Fig. 7G showed TRAF3 is correlated with BCL10 in 31 types of cancers in TCGA. Together, all these results showed TRAF3 and BCL10 can corporately participate in various biological functions and pathways, thus influencing the tumor immune microenvironment.

\section{Discussion}

Despite tremendous progresses have been made in the screen, diagnostic and therapeutic techniques and prevention for HCC, the treatment of HCC remains a bottleneck. HCC still has a highly fatality rate, as evidenced by the low 5-year survival rate[32]. Emerging studies have highlighted that the TME contributes fundamentally to initiate and maintain the pathogenesis of HCC[33]. The intricate interaction of hepatoma cells with the TME plays a critical role in regulating HCC behavior[33]. Apart from provide structural support for tumor cells, TME exert pro-tumorigenic effect via interacting dynamically with malignant cells, thus influences HCC development, metastasis and prognosis by offering inhibitory or stimulatory growth signals[34]. In this regard, treatments targeting TME and its crosstalk with hepatoma cells are underway. In addition, immune checkpoints, for instance, cytotoxic T lymphocyte protein 4 (CTLA-4), program death protein 1 (PD-1) and its ligand PD-L1 expressed on the surface of immune cells, are increased in HCC. They are associated with poorer survival by working as immunosuppressive factors in HCC microenvironment to prevent uncontrolled immune responses. Considering the immunerich TME in HCC, immune-based therapies are currently studied extensively[35]. Two forms of immunotherapy against HCC have exhibited remarkable clinical outcomes in HCC landscape: immune checkpoint inhibitors (ICls) which stimulating pre-existent anti-tumor immunity and monoclonal antibodies that directly induce T-cell dysfunction called exhaustion[36]. Our findings verified that different levels of TME have close correlations with the prognosis in HCC patients.

Thanks to advances in large-scale mutational deep-sequencing technologies, many scientists have been able to picture the mutational landscape and identify driver mutations in several solid tumors such as hepatocellular carcinoma[37]. Accumulations of genetic mutations could result in the activation of cancer-associated genes (CAGE) and contribute to hepatocarcinogenesis as evidenced by invasive tumor behaviors, such as metastatic abilities and resistance to anti-tumor drugs[38]. It has been well depicted that TP53 and CTNNB1 are the most commonly mutated CAGE in HCC and mutations of these genes are mutually independent[39]. TP53 ranked among the most frequently mutations across human sporadic tumors confirmed by genomic analysis, such as colorectal, ovarian and triple-negative breast cancer[40]. TP53 serve as "a guardian of the genome" and tumor suppressor because its role in preserving genetic stability via regulating the transcription of target genes and resulting in cell-cycle inhibition, cell aging and apoptosis in answer to DNA damage. Mutations of TP53 leading to loss-of-function of wild-type TP53 and predisposing to environmental carcinogens[41]. TP53 mutations promote oncogenesis and are related to unfavorable therapeutic responses and, hence, arise adverse prognosis in several cancer types[42]. Interestingly, the mutation rate of TP53 is very low in healthy people, while it is up to $30 \%$ in 
HCC as corroborated by the TCGA database. In HCC, the existence of TP53 mutations are significantly associated with tumor differentiation, tumor grade, Child-Pugh classification and serum AFP levels. HCC patients with TP53 mutations have poor clinical prognostic factors such as disease-free survival (DFS) and overall survival (OS), as well as high malignant behaviors and mortality of HCC[43]. Our study discovered that the overall mutation levels between two groups divided by different immune microenvironmental scores were alternated, especially TP53 mutations. According to previous analysis of the TMB impact on the survival of primary solid tumors from the TCGA database, compared with low TMB, patients with high TMB showed poorer prognosis in several cancers, and HCC is also included. Because TMB is reportedly correlated with survival rate in response to immunotherapies which aimed to kill malignant tumor cells in several cancers, and the immune microenvironment (IME) is critical for prediction of immune responses[44]. Therefore, the obscure correlation between TMB and IME in HCC requires to be explained. K-M and correlation analysis in our study uncovered for the first time that TMB is positively correlated with IME, higher levels of TMB and IME was related to worse prognosis and they predict clinical poor outcomes in HCC collaboratively.

We identified differentially expressed genes (DEGs) of HCC patients from the TCGA LIHC database on the basis of different immune infiltration and TMB levels. Additionally, functional enrichment and pathway analysis unveiled the immune-related functions of these DEGs. We further screened 89 genes associated with prognosis by Cox regression analysis. Prognostic models of 19 DGEs were also established using Lasso regression algorithm. We next generated a risk score models based on 19 hub DEGs of surviving and died patients in HCC cohorts. Finally, combining TNM staging and risk scores, a prognostic nomogram was constructed for prediction of overall survival and found that the nomogram has higher application value than the risk score model. Furthermore, AUC, C-index, DCA and calibration plot was used to demonstrate that the efficiency of constructed prognostic model was relatively good. However, before in-depth clinical practice, the prognostic data from a larger cohort is indispensable to verify these models. Besides, it is noteworthy that specific TME estimation models are essential due to the mutational environments vary among different tumor types.

B-Cell Lymphoma/Leukemia 10 (BCL10) gene is a member of BCL family, it was first detected in a translocation of mucosa-associated lymphoid tissue (MALT) lymphoma. Protein encoded by BCL10 (wildtype BCL10) could induce apoptosis and synergically forming a complex (CBM signalosome) with Caspase Recruitment Domain Family Member 11 (CRAD11) and its substrate, the MALT protein, thus initiating its downstream signaling called nuclear factor-kappa B (NF-KB). It also serves as a critical adaptor in adaptive immune responses for maintaining immune homeostasis. What's more, the CBM complex exert both cell-intrinsic and cell-extrinsic effects via influencing cells in the TME[45]. Previous studies demonstrated that mutated BCL10 still activated NF-KB but lost proapoptotic function and exhibited oncogenic activity. The dysfunction of BCL10 may lead to immunodeficiency diseases and malignancies, such as lymphoma, which mainly affects the gastric mucosa and promotes it towards a higher grade by $t(1 ; 18)(p 21 ; q 21)$ translocation $[45,46]$. Such mutations of BCL10 were discovered in other solid tumors, which suggest that BCL10 mutation may be involved in malignant transformation of human tumors. TNF Receptor Associated Factor 3 (TRAF3) gene belongs to the TRAF3 family. TRAF3 
proteins interact with CD40 (TNFRSF), which is a member of TNF receptor (TNFR) family, exerted effects on activating T-cell dependent immune responses mainly by positively modulating regulatory $T$ cells (Treg cells), consequently regulating NF-kB signal transduction and cell death[47]. Treg cells is responsible for cancer progression. In addition, TRAF3 is mainly expressed in $\mathrm{B}$ cells and restraining its survival. Inactivation mutations of TRAF3 results in NF-KB signaling activation and subsequent $\mathrm{B}$ cells survival, which enhances the tendency of malignant neoplasia[48]. TRAF3 mutations are closely related to human diseases including multiple myeloma, macroglobulinemia and cutaneous herpes infection[49]. Our study discovered for the first time that BCL10 and TRAF3 were upregulated in HCC and were higher in TP53 mutation group. What's more, TCGA database further indicated that they were associated with clinical stages and grades. However, we need to verify with in a larger sample in subsequent trails. Moreover, we explored the functions of BCL10 and TRAF3 respectively through GSEA enrichment analysis and found several co-existing functions. Considering that BCL10 and TRAF3 both associated with immune responses, ssgsea was performed and revealed that they were associated with numerous immune cells. We further uncovered the positive correlation between BCL10 and TRAF3. Therefore, we concluded that the upregulation of BCL10 and TRAF3 may affect the progression and prognosis of HCC through modulating TMB and tumor immune microenvironment.

\section{Conclusions}

Our study described that higher immune infiltration levels have associations with TMB, and they corporately predicting poor prognosis. Our study may contribute to the unveiling of predictive roles of BCL10 and TRAF3 expression in evaluating hepatic carcinogenesis and clinical prognosis for patients with HCC, which may function by regulating TMB and tumor immune microenvironment.

\section{Abbreviations}

$\mathrm{HCC}$

Hepatocellular carcinoma; TMB:Tumor mutational burden; DEGs:Differential expression genes; TME:Tumor microenvironment; BCL10:B-Cell Lymphoma/Leukemia 10; TRAF3:TNF Receptor Associated Factor 3; ICl:Immune checkpoint inhibitor; AHNTU:affiliated hospital of Nantong University; IHC:Immunohistochemistry; qRT-PCR:quantitative real time-polymerase chain reaction; TCGA:The Cancer Genome Atlas; ssGSEA:Single-Sample Gene Set Enrichment Analysis; GO:Gene ontology; KEGG:Kyoto Encyclopedia of Genes and Genomes; NRI:Net reclassification index; DCA:Decision Curve Analysis; CTLA4:cytotoxic T lymphocyte protein 4; PD-1:program death protein 1; CAGE:cancer-associated genes

\section{Declarations}

\section{Ethics approval and consent to participate}

The study was approved by the ethics committee of the affiliated hospital of Nantong University (AHNTU), written informed consent was obtained from all patients in our study. 


\section{Consent for publication}

Not applicable.

\section{Availability of data and materials}

The anonymized data used and/or analyzed during the current study are available from the corresponding author upon reasonable request.

\section{Competing interests}

The authors declare that they have no competing interests.

\section{Funding}

None

\section{Authors' contributions}

$\mathrm{CHL}$ and WH conceived and designed the whole study, JC is responsible for drafting the manuscript, SZW carried out acquisition, analyses and interpretation of the data, SJG, RJ and YYC involved in critically revising the manuscript for important intellectual content. All authors reviewed and approved the final manuscript.

\section{Acknowledgements}

This study was supported by the National Natural Science Foundation of China (NO. 82070624).

\section{References}

1. Bray F, Ferlay J, Soerjomataram I, Siegel R, Torre L, Jemal A. Global cancer statistics 2018 : GLOBOCAN estimates of incidence and mortality worldwide for 36 cancers in 185 countries. Cancer J Clin. 2018;68(6):394-424.

2. Fouad Y, Lazarus J, Negro F, Peck-Radosavljevic M, Sarin S, Ferenci P, Esmat G, Ghazinian H, Nakajima A, Silva M, et al. MAFLD considerations as a part of the global hepatitis $C$ elimination effort: an international perspective. Alimentary pharmacology \& therapeutics; 2021.

3. Singal A, Lampertico P, Nahon P. Epidemiology and surveillance for hepatocellular carcinoma: New trends. Journal of hepatology. 2020;72(2):250-61.

4. Takeda A, Sanuki N, Tsurugai Y, Iwabuchi S, Matsunaga K, Ebinuma H, Imajo K, Aoki Y, Saito H, Kunieda E. Phase 2 study of stereotactic body radiotherapy and optional transarterial chemoembolization for solitary hepatocellular carcinoma not amenable to resection and radiofrequency ablation. Cancer. 2016;122(13):2041-9. 
5. Forner A, Reig M, Bruix J. Hepatocellular carcinoma. Lancet. 2018;391(10127):1301-14.

6. Rebouissou S, Franconi A, Calderaro J, Letouzé E, Imbeaud S, Pilati C, Nault J, Couchy G, Laurent A, Balabaud C, et al. Genotype-phenotype correlation of CTNNB1 mutations reveals different B-catenin activity associated with liver tumor progression. Hepatology. 2016;64(6):2047-61.

7. Yarchoan M, Hopkins A, Jaffee E. Tumor Mutational Burden and Response Rate to PD-1 Inhibition. N Engl J Med. 2017;377(25):2500-1.

8. Chan T, Yarchoan M, Jaffee E, Swanton C, Quezada S, Stenzinger A, Peters S. Development of tumor mutation burden as an immunotherapy biomarker: utility for the oncology clinic. Annals of oncology: official journal of the European Society for Medical Oncology. 2019;30(1):44-56.

9. Panda A, Betigeri A, Subramanian K, Ross J, Pavlick D, Ali S, Markowski P, Silk A, Kaufman H, Lattime E, et al: Identifying a Clinically Applicable Mutational Burden Threshold as a Potential Biomarker of Response to Immune Checkpoint Therapy in Solid Tumors. JCO precision oncology 2017, 2017.

10. Cao D, Xu H, Xu X, Guo T, Ge W. High tumor mutation burden predicts better efficacy of immunotherapy: a pooled analysis of 103078 cancer patients. Oncoimmunology. 2019;8(9):e1629258.

11. Wu H, Wang Z, Zhao Q, Chen D, He M, Yang L, Wang Y, Jin Y, Ren C, Luo H, et al. Tumor mutational and indel burden: a systematic pan-cancer evaluation as prognostic biomarkers. Annals of translational medicine. 2019;7(22):640.

12. Kazdal D, Endris V, Allgäuer M, Kriegsmann M, Leichsenring J, Volckmar A, Harms A, Kirchner M, Kriegsmann K, Neumann O, et al. Spatial and Temporal Heterogeneity of Panel-Based Tumor Mutational Burden in Pulmonary Adenocarcinoma: Separating Biology From Technical Artifacts. Journal of thoracic oncology: official publication of the International Association for the Study of Lung Cancer. 2019;14(11):1935-47.

13. Hughes R, Simons B, Khan H, Miller R, Kugler V, Torquato S, Theodros D, Haffner M, Lotan T, Huang J, et al. Asporin Restricts Mesenchymal Stromal Cell Differentiation, Alters the Tumor Microenvironment, and Drives Metastatic Progression. Cancer research. 2019;79(14):3636-50.

14. Peng Y, Liu C, Li M, Li W, Zhang M, Jiang X, Chang Y, Liu L, Wang F, Zhao Q. Identification of a prognostic and therapeutic immune signature associated with hepatocellular carcinoma. Cancer cell international. 2021;21(1):98.

15. Li X, Wenes M, Romero P, Huang S, Fendt S, Ho P. Navigating metabolic pathways to enhance antitumour immunity and immunotherapy. Nature reviews Clinical oncology. 2019;16(7):425-41.

16. Ribas A, Wolchok J. Cancer immunotherapy using checkpoint blockade. Science. 2018;359(6382):1350-5.

17. High TMB. Predicts Immunotherapy Benefit. Cancer discovery. 2018;8(6):668.

18. Colaprico A, Silva T, Olsen C, Garofano L, Cava C, Garolini D, Sabedot T, Malta T, Pagnotta S, Castiglioni I, et al. TCGAbiolinks: an R/Bioconductor package for integrative analysis of TCGA data. Nucleic acids research. 2016;44(8):e71. 
19. Mayakonda A, Lin D, Assenov Y, Plass C, Koeffler H. Maftools: efficient and comprehensive analysis of somatic variants in cancer. Genome research. 2018;28(11):1747-56.

20. Yi M, Nissley D, McCormick F, Stephens R. ssGSEA score-based Ras dependency indexes derived from gene expression data reveal potential Ras addiction mechanisms with possible clinical implications. Scientific reports. 2020;10(1):10258.

21. Chen B, Khodadoust M, Liu C, Newman A, Alizadeh A: Profiling Tumor Infiltrating Immune Cells with CIBERSORT. Methods in molecular biology (Clifton, NJ) 2018, 1711:243-259.

22. Rasero J, Diez I, Cortes J, Marinazzo D, Stramaglia S. Connectome sorting by consensus clustering increases separability in group neuroimaging studies. Network neuroscience (Cambridge Mass). 2019;3(2):325-43.

23. Chalmers Z, Connelly C, Fabrizio D, Gay L, Ali S, Ennis R, Schrock A, Campbell B, Shlien A, Chmielecki $J$, et al. Analysis of 100,000 human cancer genomes reveals the landscape of tumor mutational burden. Genome medicine. 2017;9(1):34.

24. Love M, Huber W, Anders S: Moderated estimation of fold change and dispersion for RNA-seq data with DESeq2. Genome biology 2014, 15(12):550.

25. Gene Ontology Consortium. : going forward. Nucleic acids research. 2015;43:D1049-56.

26. Subramanian A, Tamayo P, Mootha V, Mukherjee S, Ebert B, Gillette M, Paulovich A, Pomeroy S, Golub T, Lander $E$, et al. Gene set enrichment analysis: a knowledge-based approach for interpreting genome-wide expression profiles. Proc Natl Acad Sci USA. 2005;102(43):15545-50.

27. Engebretsen S, Bohlin J. Statistical predictions with glmnet. Clinical epigenetics. 2019;11(1):123.

28. Sing T, Sander O, Beerenwinkel N, Lengauer T. ROCR: visualizing classifier performance in R. Bioinformatics. 2005;21(20):3940-1.

29. Park S. Nomogram: An analogue tool to deliver digital knowledge. J Thorac Cardiovasc Surg. 2018;155(4):1793.

30. Chen Y, Zhang X, Xu C, Zhou D, Chen J, Liu Z, Sun Y, Huang W, Qu L. MCCC2 promotes HCC development by supporting leucine oncogenic function. Cancer cell international. 2021;21(1):22.

31. Ito K, Murphy D. Application of ggplot2 to Pharmacometric Graphics. CPT: pharmacometrics systems pharmacology. 2013;2:e79.

32. Zhou Y, Ding J, Qin Z, Wang Y, Zhang J, Jia K, Wang Y, Zhou H, Wang F, Jing X. Predicting the survival rate of patients with hepatocellular carcinoma after thermal ablation by nomograms. Annals of translational medicine. 2020;8(18):1159.

33. Wu Q, Zhou L, Lv D, Zhu X, Tang H. Exosome-mediated communication in the tumor microenvironment contributes to hepatocellular carcinoma development and progression. J Hematol Oncol. 2019;12(1):53.

34. Ilkhani K, Bastami M, Delgir S, Safi A, Talebian S, Alivand M. The Engaged Role of Tumor Microenvironment in Cancer Metabolism: Focusing on Cancer-Associated Fibroblast and Exosome Mediators. Anti-cancer Agents Med Chem. 2021;21(2):254-66. 
35. Greten T, Lai C, Li G, Staveley-O'Carroll K. Targeted and Immune-Based Therapies for Hepatocellular Carcinoma. Gastroenterology. 2019;156(2):510-24.

36. Chan K, Bass A. Autoimmune complications of immunotherapy: pathophysiology and management. BMJ. 2020;369:m736.

37. Schulze K, Nault J, Villanueva A. Genetic profiling of hepatocellular carcinoma using next-generation sequencing. Journal of hepatology. 2016;65(5):1031-42.

38. Lin C, Yuan G, Hu Z, Zeng Y, Qiu X, Yu H, He S. Bioinformatics analysis of the interactions among IncRNA, miRNA and mRNA expression, genetic mutations and epigenetic modifications in hepatocellular carcinoma. Mol Med Rep. 2019;19(2):1356-64.

39. Kawai-Kitahata F, Asahina Y, Tanaka S, Kakinuma S, Murakawa M, Nitta S, Watanabe T, Otani S, Taniguchi M, Goto F, et al. Comprehensive analyses of mutations and hepatitis B virus integration in hepatocellular carcinoma with clinicopathological features. Journal of gastroenterology. 2016;51(5):473-86.

40. Hainaut P, Pfeifer G. Somatic TP53 Mutations in the Era of Genome Sequencing. Cold Spring Harbor perspectives in medicine 2016, 6(11).

41. Lim Y, Lim T, Chan Y, Song A, Yeo B, Vojtesek B, Coomber D, Rajagopal G, Lane D. The p53 knowledgebase: an integrated information resource for $p 53$ research. Oncogene. 2007;26(11):151721.

42. Yang C, Huang X, Li Y, Chen J, Lv Y, Dai S. Prognosis and personalized treatment prediction in TP53mutant hepatocellular carcinoma: an in silico strategy towards precision oncology. Briefings in bioinformatics 2020.

43. Harding J, Nandakumar S, Armenia J, Khalil D, Albano M, Ly M, Shia J, Hechtman J, Kundra R, El Dika I, et al. Prospective Genotyping of Hepatocellular Carcinoma: Clinical Implications of NextGeneration Sequencing for Matching Patients to Targeted and Immune Therapies. Clinical cancer research: an official journal of the American Association for Cancer Research. 2019;25(7):2116-26.

44. Zheng C, Zheng L, Yoo J, Guo H, Zhang Y, Guo X, Kang B, Hu R, Huang J, Zhang Q, et al. Landscape of Infiltrating T Cells in Liver Cancer Revealed by Single-Cell Sequencing. Cell. 2017;169(7):134256.e1316.

45. Turvey S, Durandy A, Fischer A, Fung S, Geha R, Gewies A, Giese T, Greil J, Keller B, McKinnon M, et al. The CARD11-BCL10-MALT1 (CBM) signalosome complex: Stepping into the limelight of human primary immunodeficiency. J Allergy Clin Immunol. 2014;134(2):276-84.

46. Kuo S, Chen L, Yeh K, Wu M, Hsu H, Yeh P, Mao T, Chen C, Doong S, Lin J, et al. Nuclear expression of BCL10 or nuclear factor kappa B predicts Helicobacter pylori-independent status of early-stage, highgrade gastric mucosa-associated lymphoid tissue lymphomas. Journal of clinical oncology: official journal of the American Society of Clinical Oncology. 2004;22(17):3491-7.

47. Aronchik I, Bjeldanes L, Firestone G. Direct inhibition of elastase activity by indole-3-carbinol triggers a CD40-TRAF regulatory cascade that disrupts NF-kappaB transcriptional activity in human breast cancer cells. Cancer research. 2010;70(12):4961-71. 
48. Lin W, Yi Z, Stunz L, Maine C, Sherman L, Bishop G. The adaptor protein TRAF3 inhibits interleukin-6 receptor signaling in B cells to limit plasma cell development. Sci Signal. 2015;8(392):ra88.

49. Braggio E, Keats J, Leleu X, Van Wier S, Jimenez-Zepeda V, Valdez R, Schop R, Price-Troska T, Henderson K, Sacco A, et al. Identification of copy number abnormalities and inactivating mutations in two negative regulators of nuclear factor-kappaB signaling pathways in Waldenstrom's macroglobulinemia. Cancer research. 2009;69(8):3579-88.

\section{Figures}


A

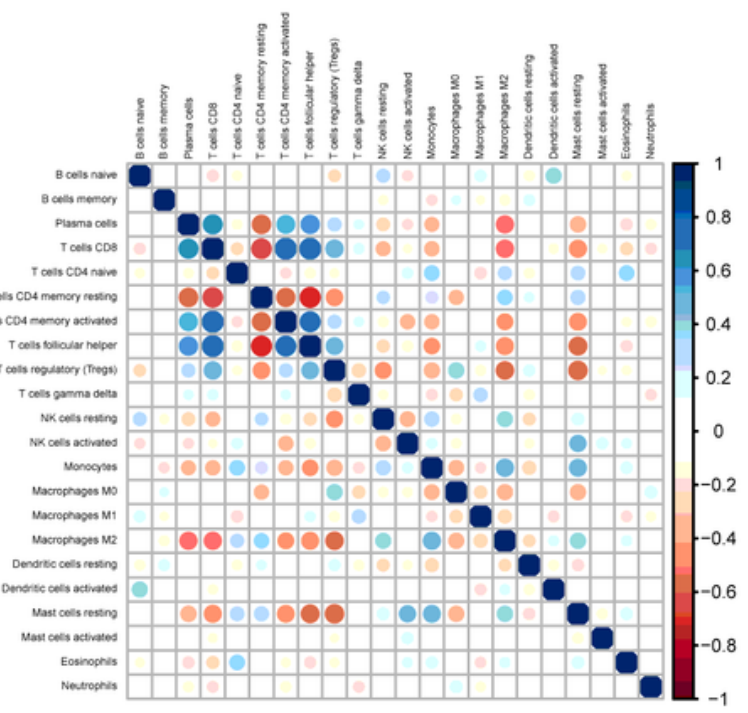

C

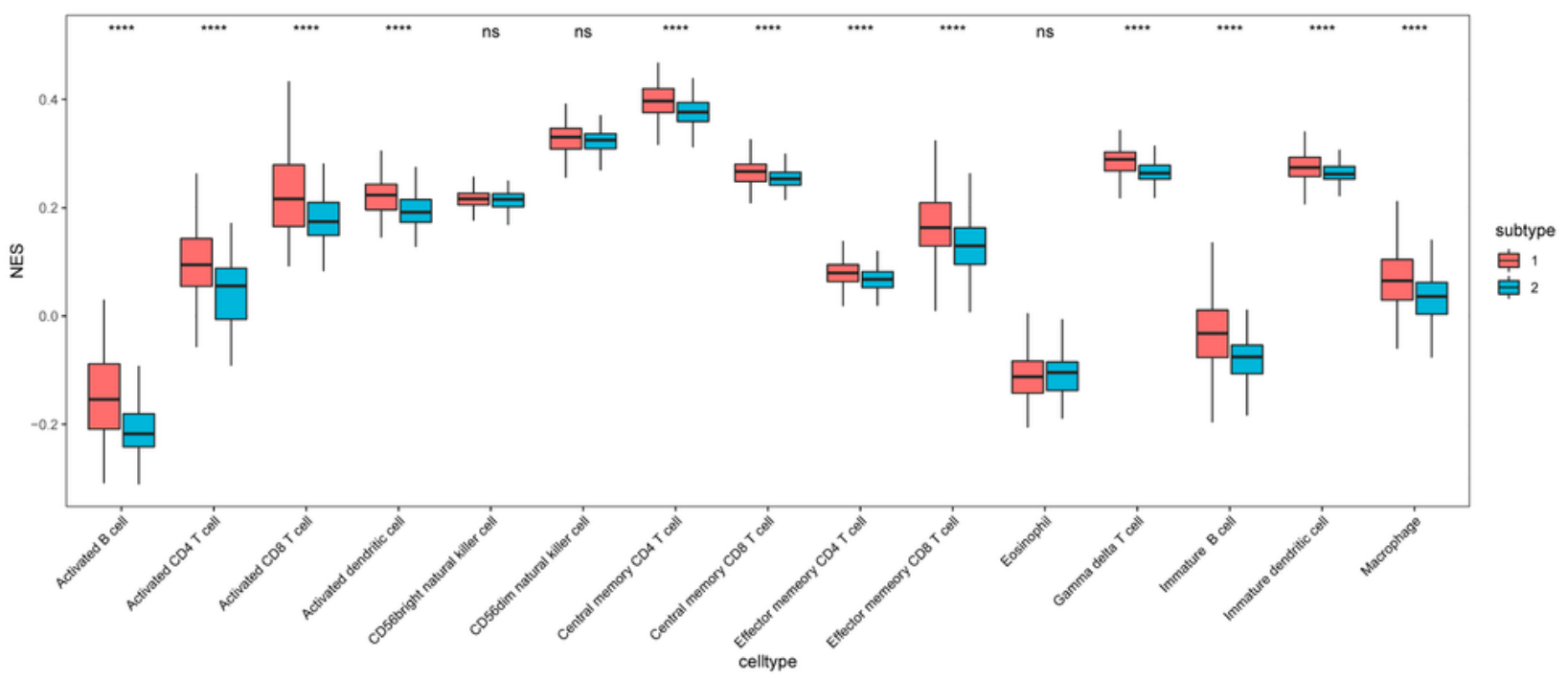

B

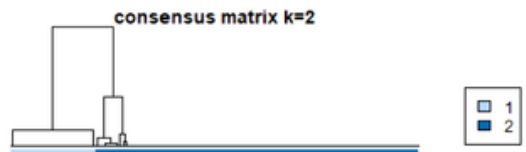

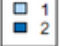

D

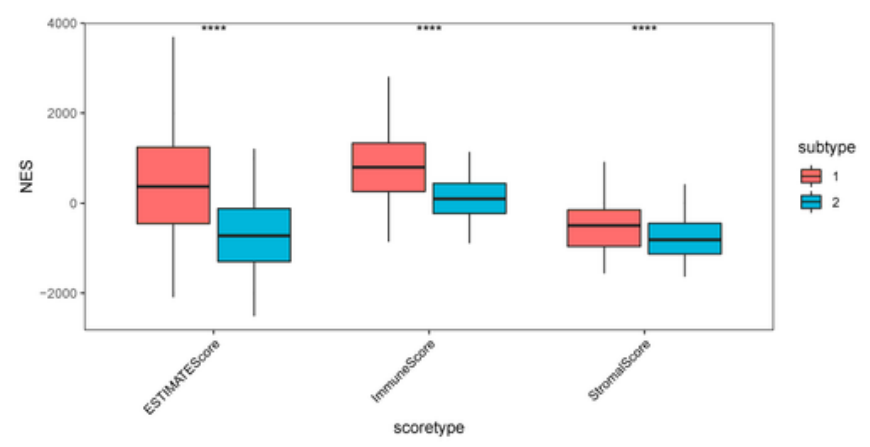

E

Strata - Group $=1$ - Group $=2$
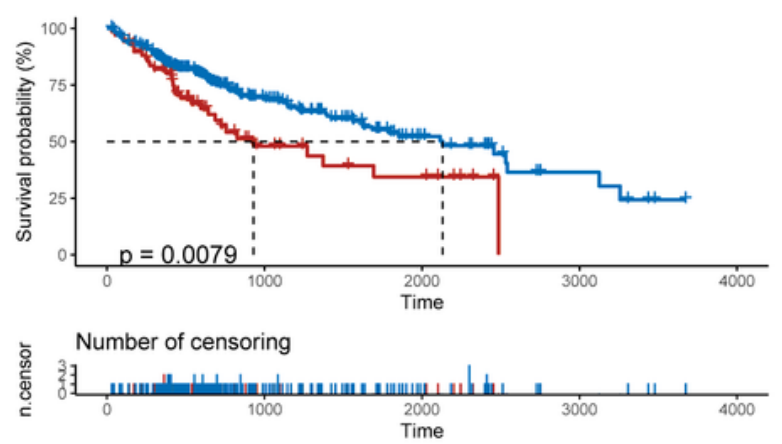

\section{Figure 1}

Immune infiltration landscape of HCC patients in TCGA. a Immune cell interaction in HCC was plotted by the correlation coefficient heatmap. b 374 samples were divided into two subtypes by consensus cluster analysis by the optimal cluster number. c ssGSEA analysis displayed associations of 2 immune clusters (ICR) with immune cells. $d$ Estimate scores, immune scores and stromal scores of ICR1 and ICR2 are 
obtained with the ESTIMATE algorithm. e Overall survival (OS) of ICR1 and ICR2 group patients are visualized using Kaplan-Meier survival analysis.

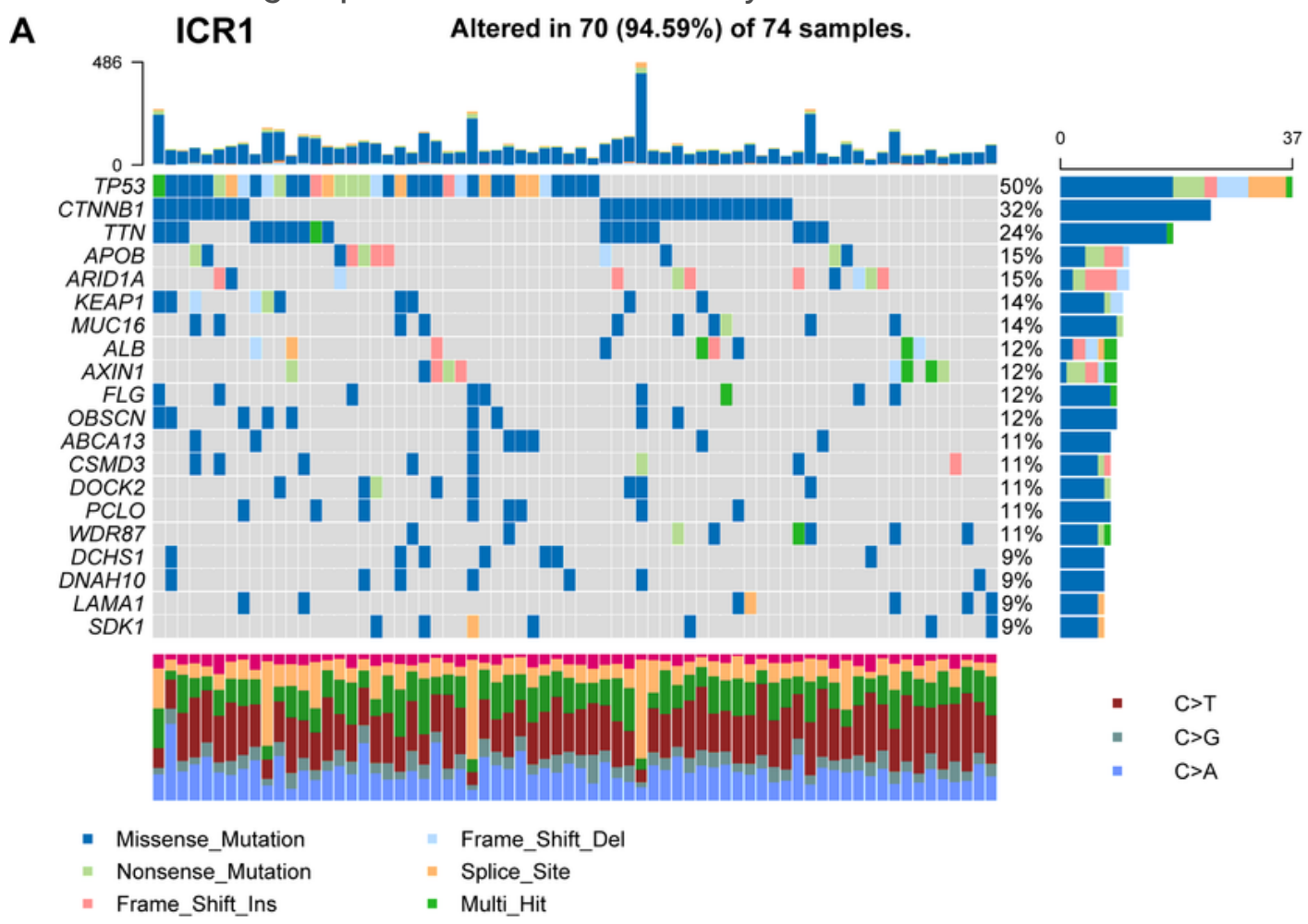

B

ICR2

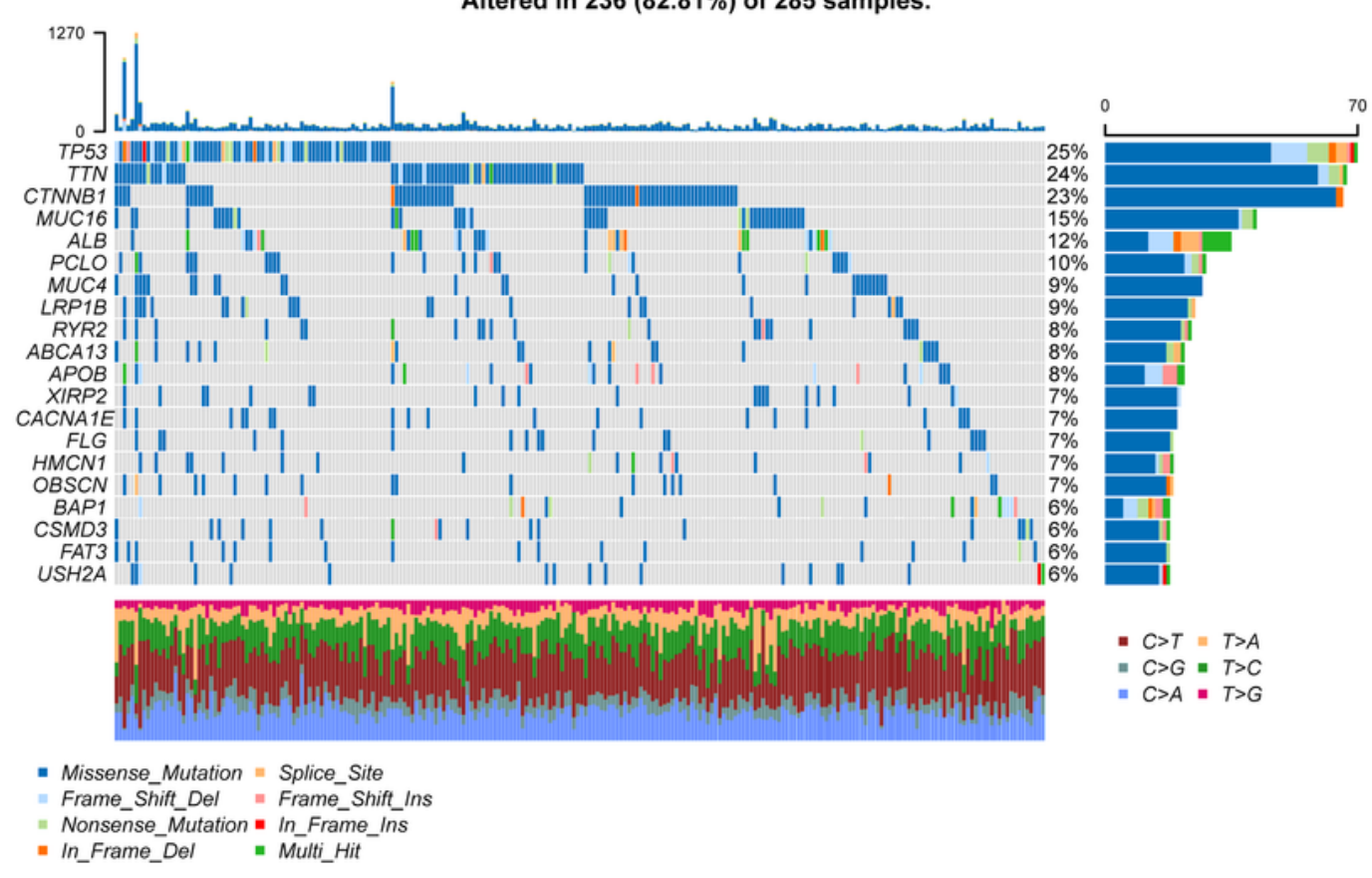

Figure 2

Comparison of HCC mutation profiles. Waterfall plot exhibited mutation particulars of each gene in the ICR1 (a) and ICR2 (b) group. The different colored notes at the bottom symbolized different mutation types. 
A

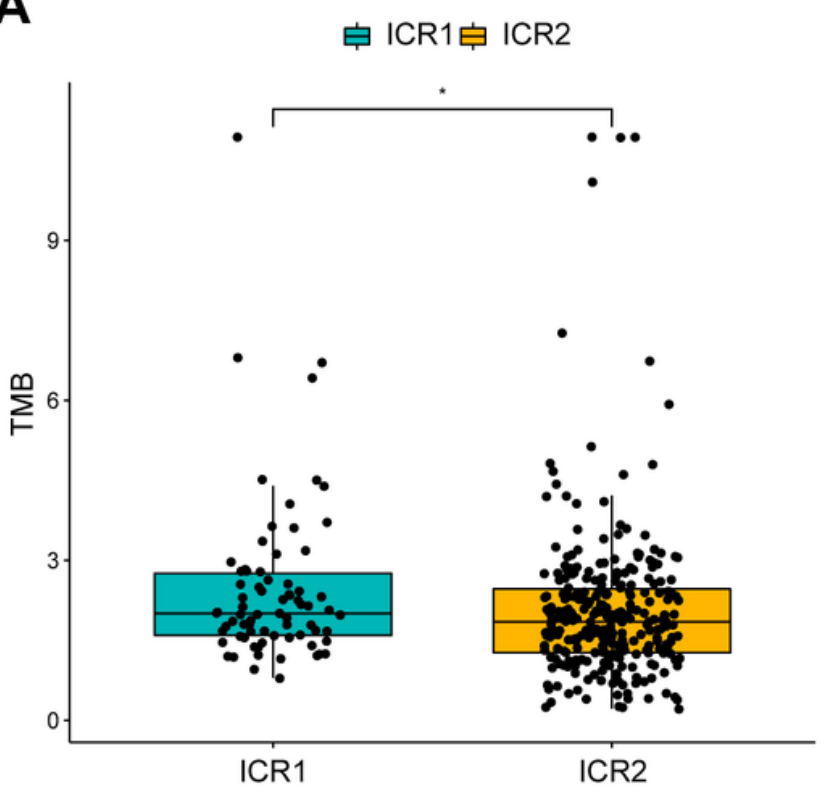

C
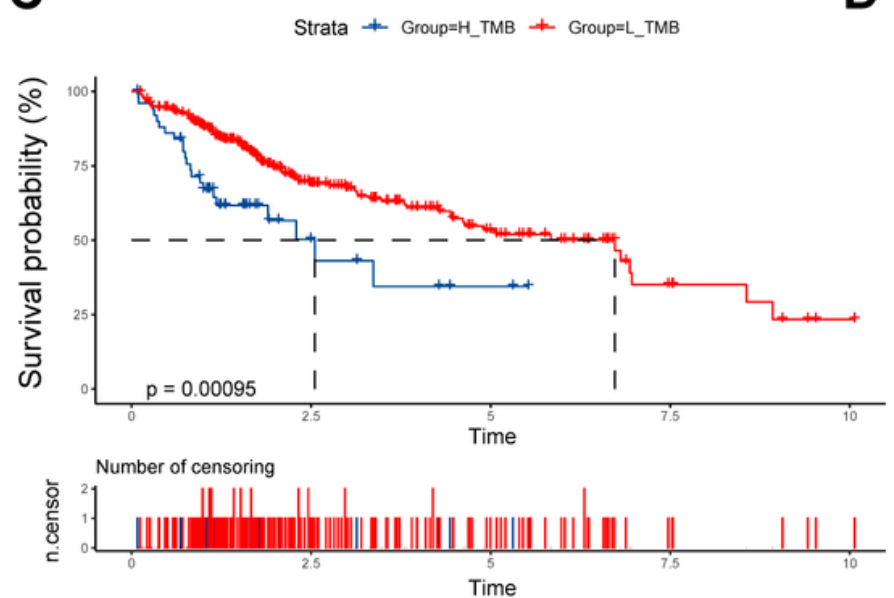

B

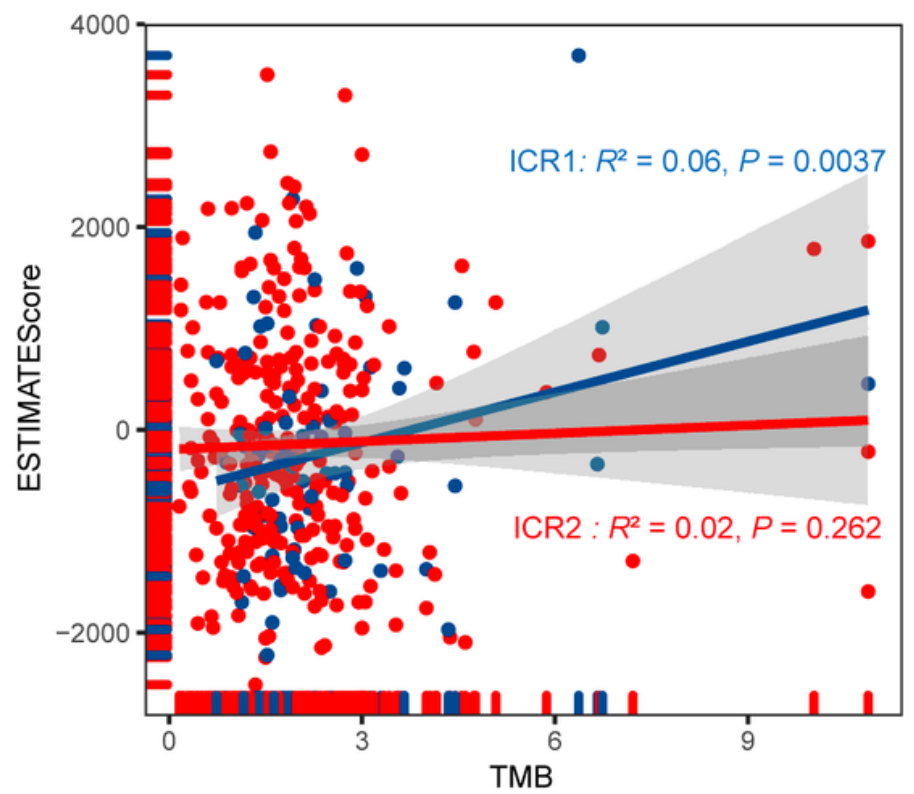

group

- ICR1

- ICR2
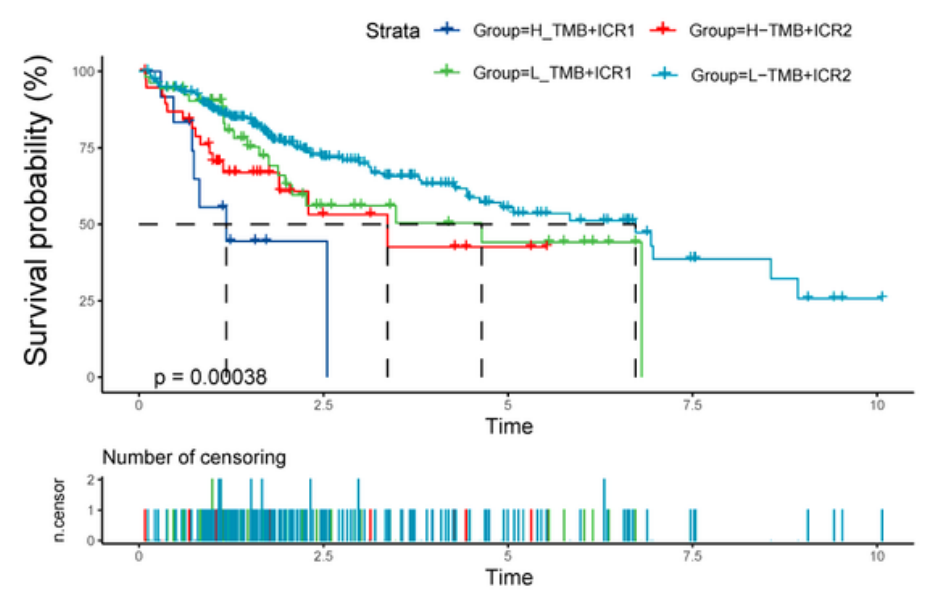

\section{Figure 3}

The Correlation between the immune infiltration and Tumor mutation burden in HCC. a Tumor mutation burden (TMB) of ICR1 and ICR2 group were assessed. b Correlation analysis of the Estimate score and TMB of ICR1 and ICR2 group was exhibited. c Kaplan-Meier survival curves of the overall survival (OS) in high and low TMB patients. d Kaplan-Meier survival curves displayed survival rate between subgroups (High TMB \& ICR1 group versus High TMB \& ICR2 group; Low TMB \& ICR1 score group versus Low TMB \& ICR2 group). 
A

B

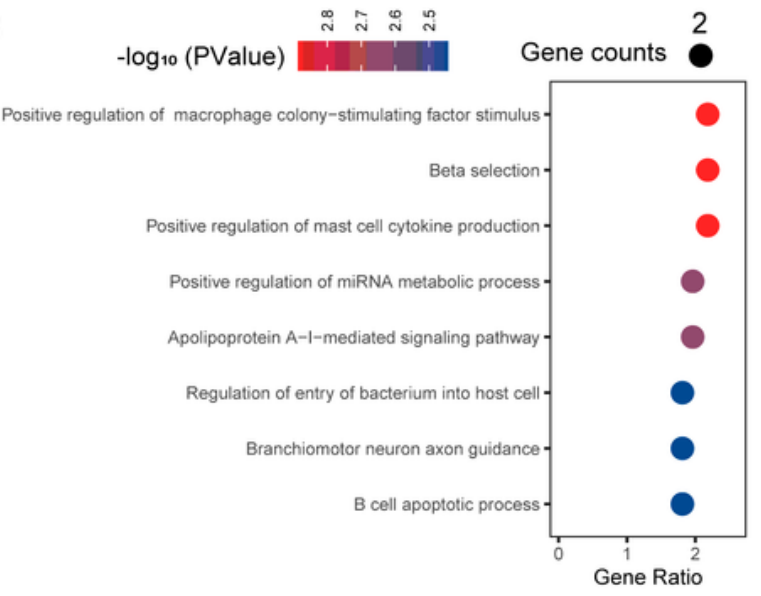

$\mathrm{BP}$
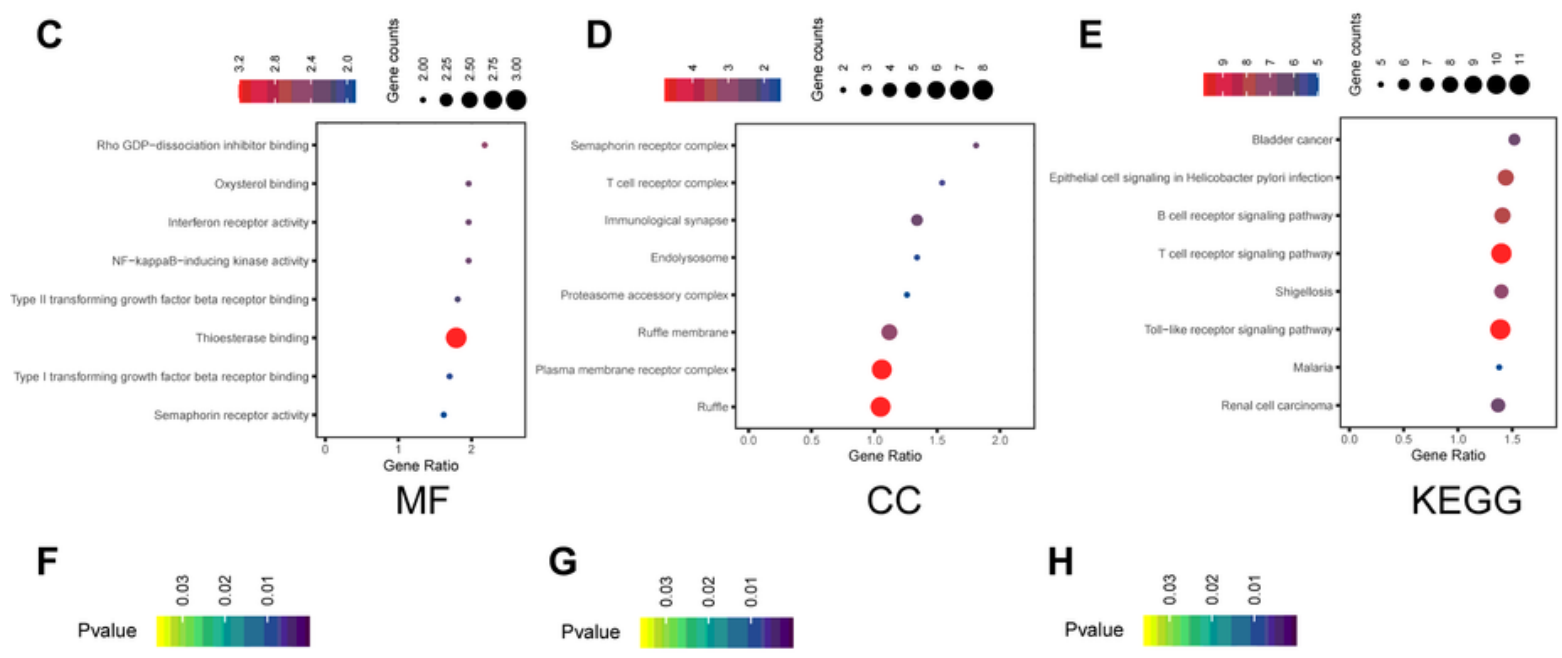

MF

$\mathrm{CC}$

KEGG
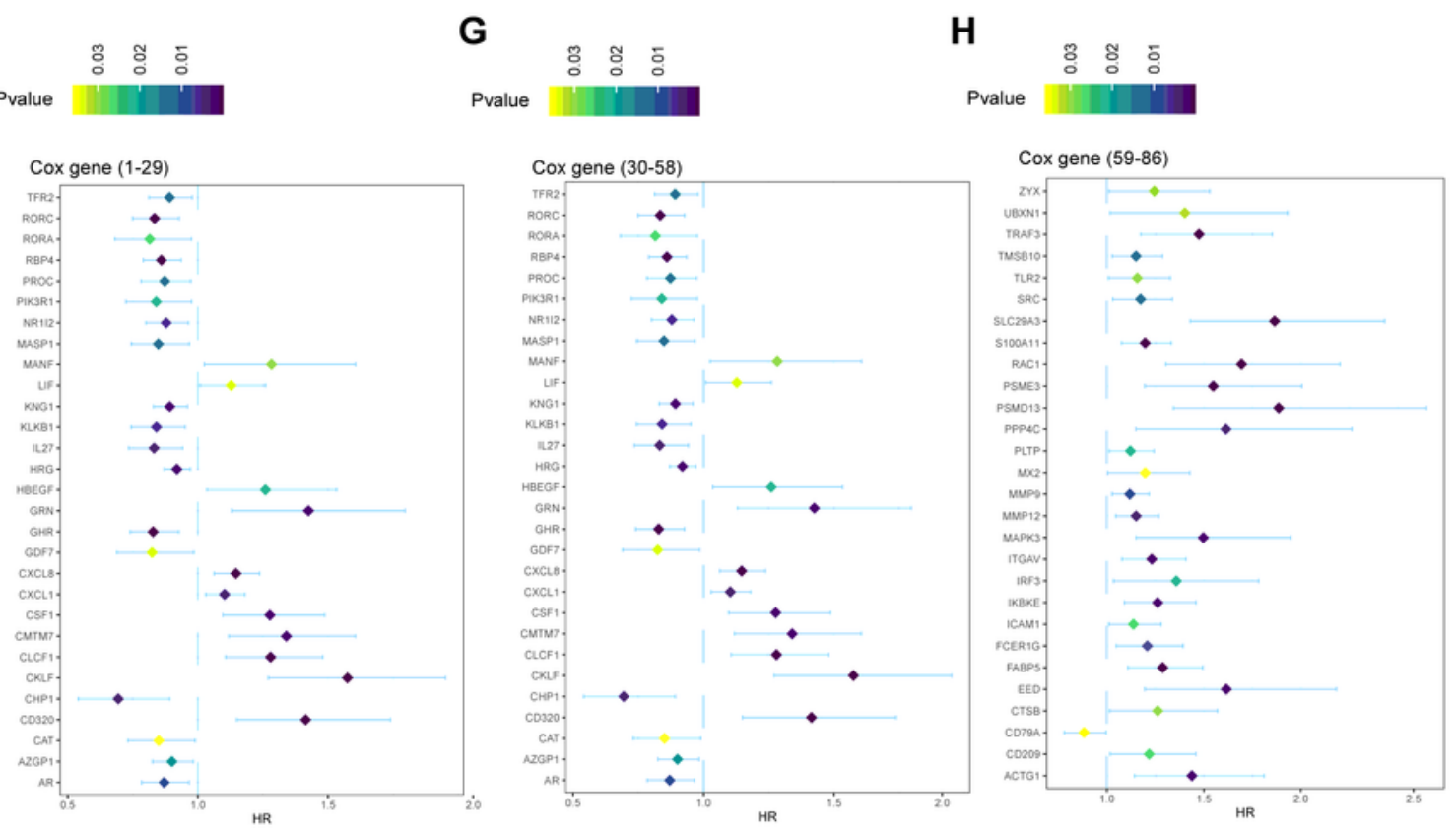

\section{Figure 4}

Differential expressed genes (DEGs) based on immune and TMB score in HCC. a Intersection map of 369 DEGs involcing TMB score and Immune score. b, c, d Gene Ontology (GO) annotations of biological processes (BP), molecular functions (MF) and cellular components (CC) of 369 DEGs. e Bubble chart showed analysis of Kyoto Encyclopedia of Genes and Genomes (KEGG) pathway for 369 DEGs. f, g, h 
Hazard ratio in the univariate Cox regression analysis of 89 significant DEGs was visualized in the forest plot.

A

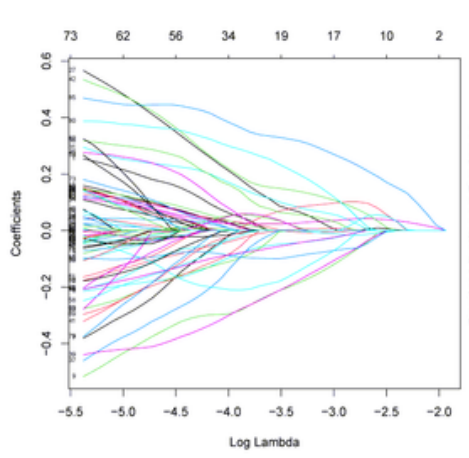

D

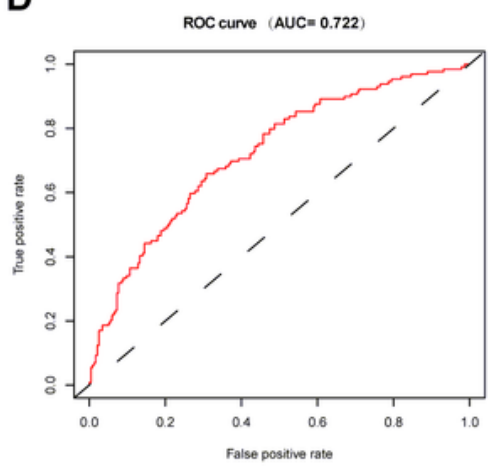

B

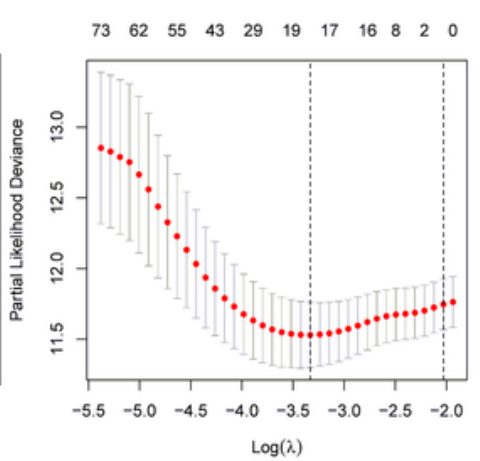

$\mathbf{F}$

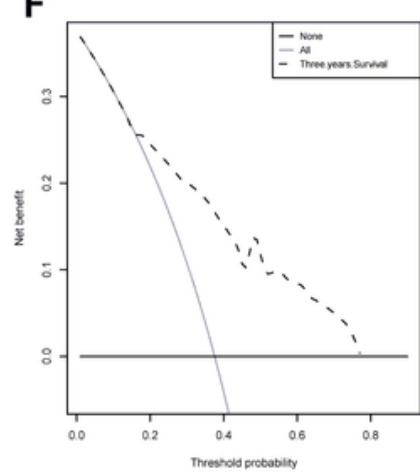

C

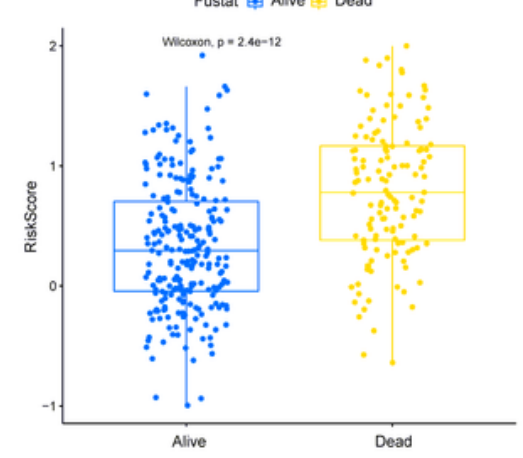

G

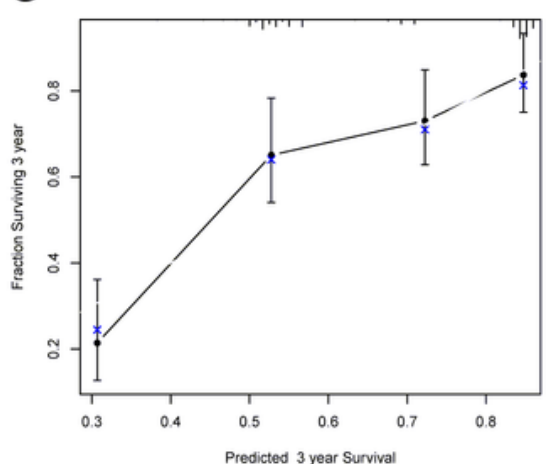

E

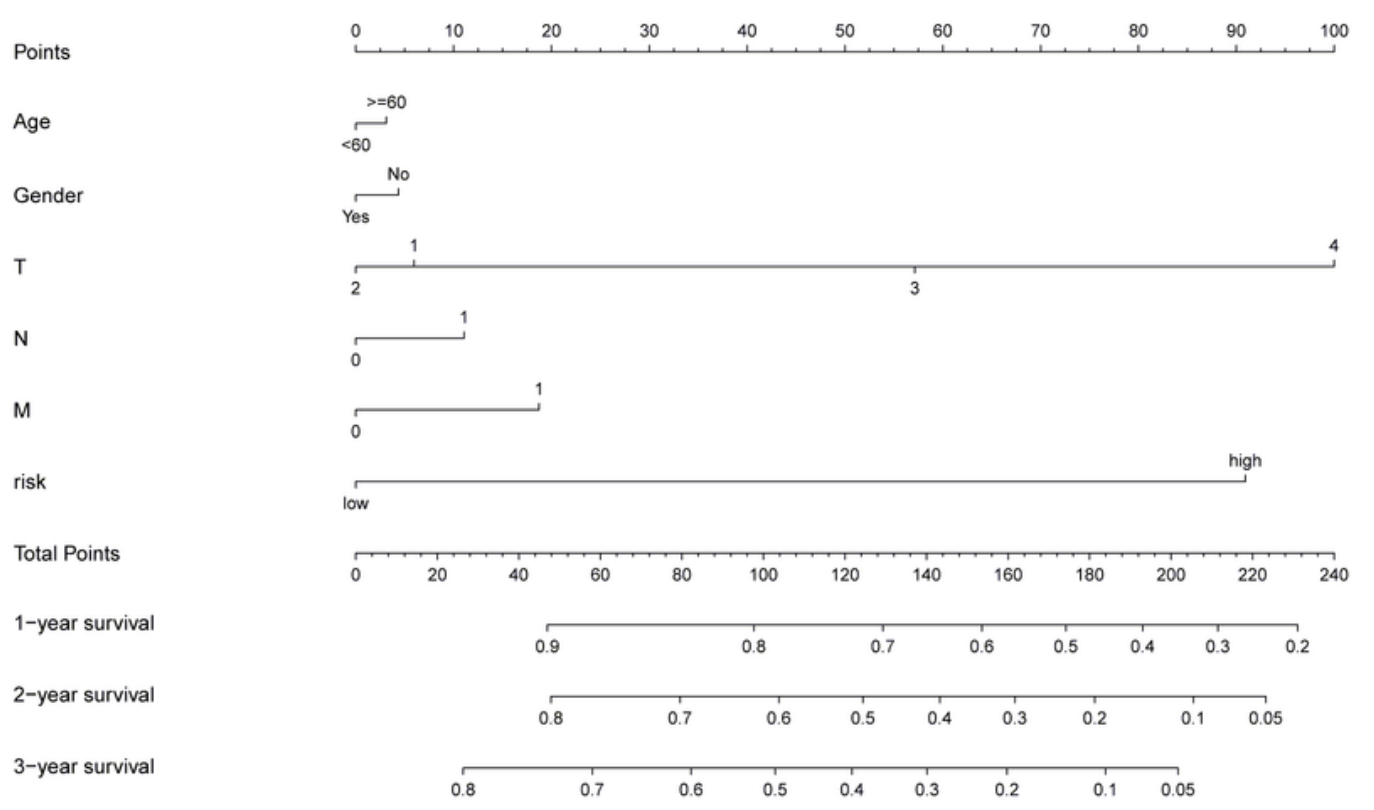

\section{Figure 5}

Construction of the combined prognostic risk profile for HCC. a A coefficient profile plot determined by the minimum likelihood deviance was generated. b Nineteen DEGs and corresponding coefficients were exhibited by cross-validation. c The risk score of dead and alive patients. $d$ Receiver operating 
characteristic (ROC) curve was generated for evaluating the Prediction accuracy. The AUC index of ROC curve was shown in the bracket (0.722). e On the basis of age, gender, TNM stage and risk score, a nomogram was constructed to estimate 1-, 2-, 3-year survival rate for HCC. f The DCA plots reflected the net benefit of the nomogram. $g$ Calibration plots further verified the predictive ability of our model.

B

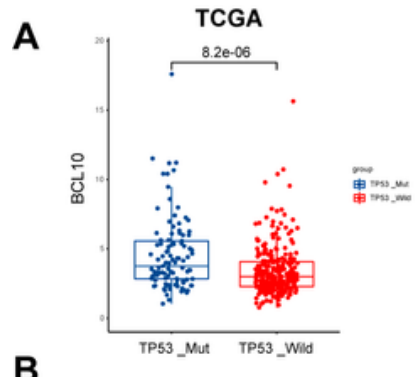

G

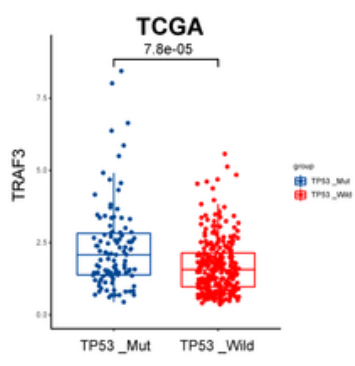

$\mathrm{HCC}$

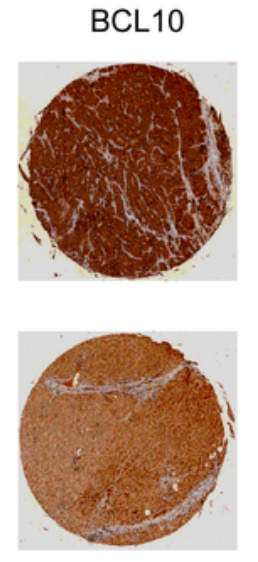

H

TRAF6

$\mathrm{HCC}$

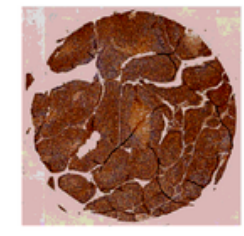

Normal

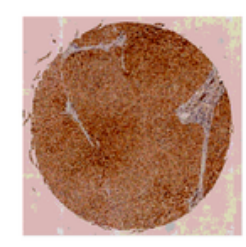

c

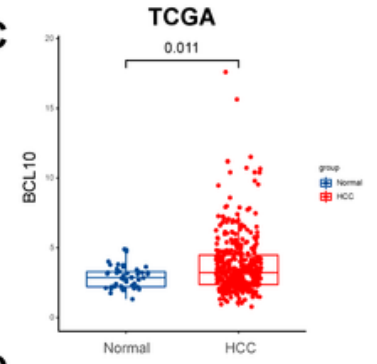

D

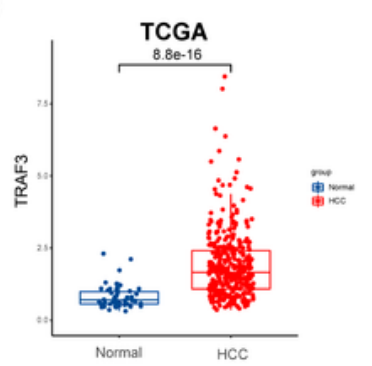

E

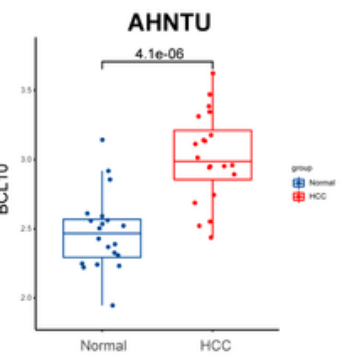

F

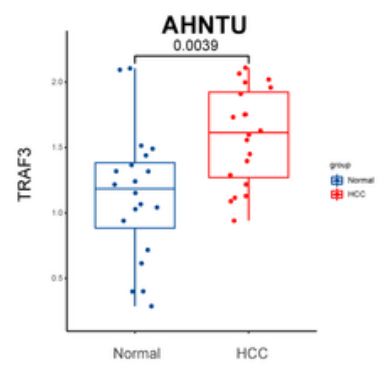

I

\begin{tabular}{|c|c|c|c|c|c|}
\hline $\mathrm{HCC}$ & Status & Stage & M & $\mathrm{N}$ & $\mathrm{T}$ \\
\hline $\begin{array}{l}\text { High BCL10 } \\
\qquad(n=117)\end{array}$ & & & & & \\
\hline $\begin{array}{l}\text { Low BCL10 } \\
\quad(n=117)\end{array}$ & & & & & \\
\hline$P$ value & $p=0.008$ & $p=0.0075$ & $p=0.36$ & $p=0.36$ & $p=0.0076$ \\
\hline
\end{tabular}

J

\begin{tabular}{|c|c|c|c|c|c|}
\hline $\mathrm{HCC}$ & Status & Stage & $M$ & $\mathrm{~N}$ & $\mathrm{~T}$ \\
\hline $\begin{array}{l}\text { High TRAF3 } \\
\quad(n=117)\end{array}$ & & & & & \\
\hline $\begin{array}{l}\text { Low TRAF3 } \\
\quad(n=117)\end{array}$ & & & & & \\
\hline$P$ value & $p=0.093$ & $p=0.044$ & $p=0.37$ & $p=1$ & $p=0.14$ \\
\hline
\end{tabular}

Figure 6 

high BCL10 and TRAF3 expression with the status, Ajcc stage and TMN stage.

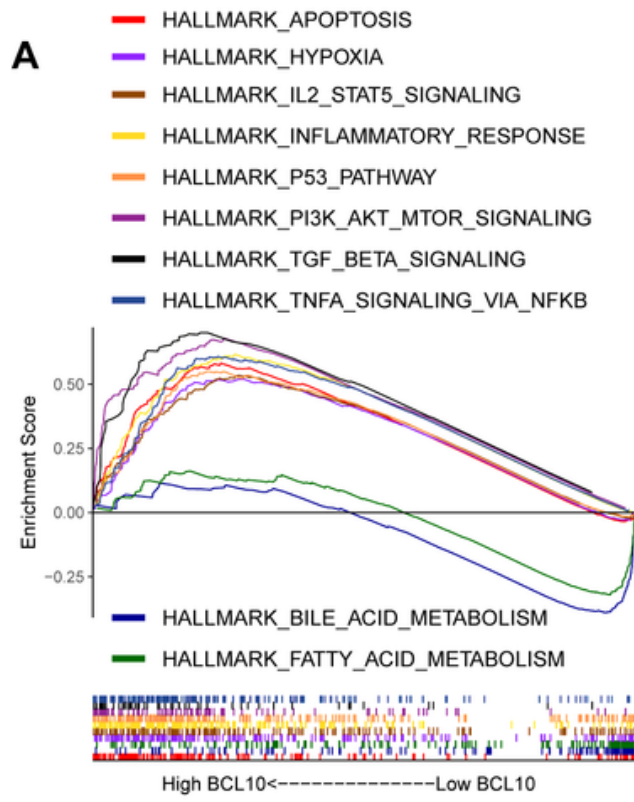

C

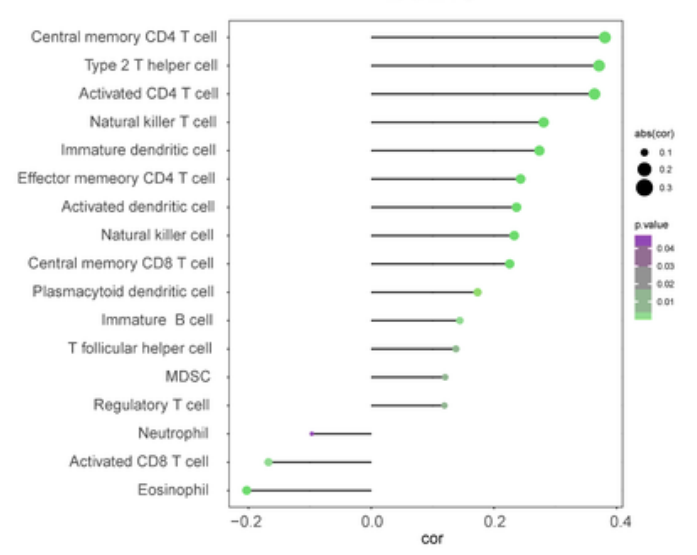

E
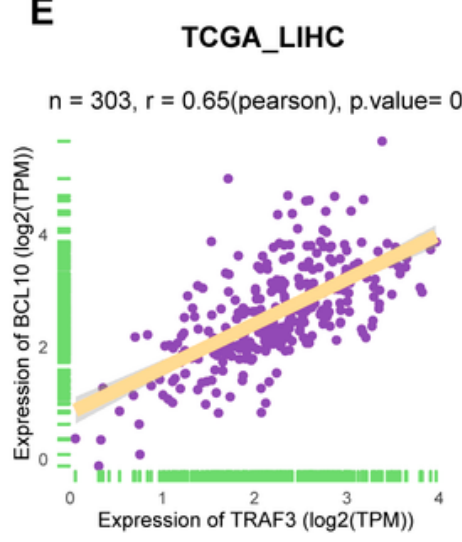

Figure 7

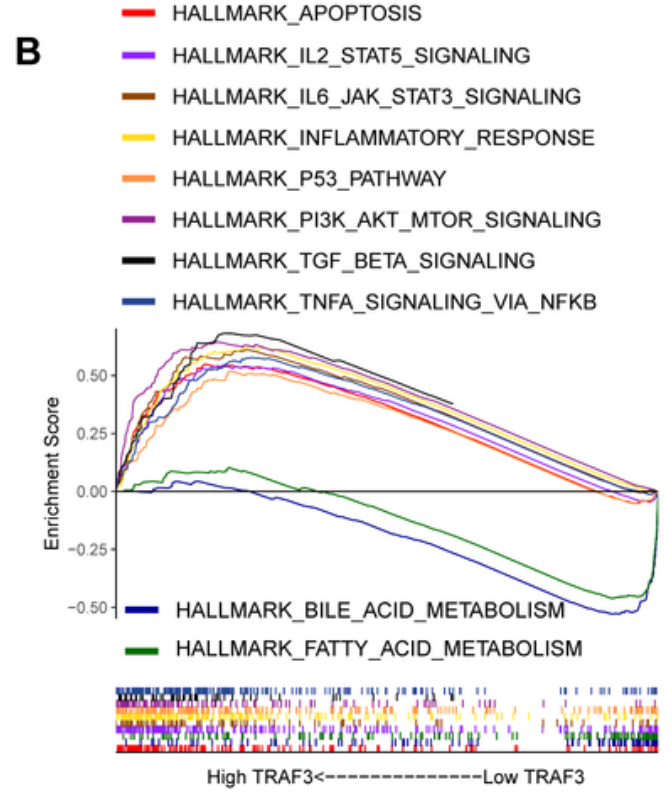

D

TRAF3

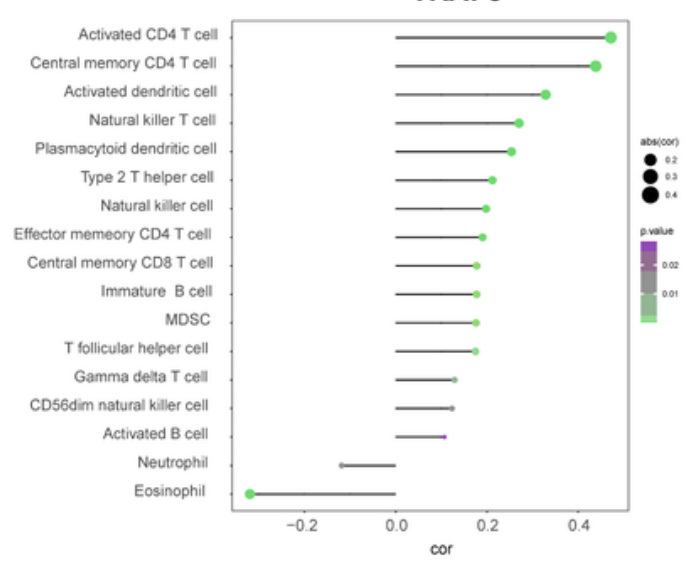

G

TCGA_panCancer

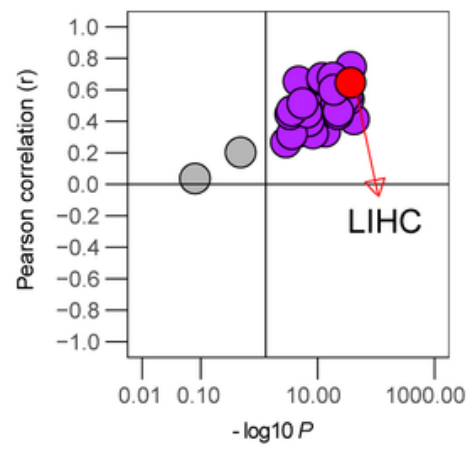


Functional enrichment and correlation analysis of BCL10 and TRAF3. a, b GSEA analysis showing the commonly enriched functions of BCL10 and TRAF3. $c$, $d$ the correlation of BCL10 and TRAF3 expression with immune cells were listed in SSGSEA plots. e Pearson test showing that BCL10 is positively correlated with TRAF3 in HCC. $f, g$ Positive correlation of BCL10 and TRAF3 in pan cancers was validated in TCGA cohort. 